\title{
https://doi.org/10.46813/2021-134-128 \\ ENERGY LOSSES OF A POINT MAGNETIC DIPOLE DUE TO INTERACTION WITH A MAGNETIZED PLASMA CYLINDER
}

\author{
Yu.O. Averkov ${ }^{1,2}$, Yu.V. Prokopenko ${ }^{1,3}$, A.A. Shmat'ko', V.M. Yakovenko' \\ ${ }^{1}$ A. Ya. Usikov Institute for Radiophysics and Electronics of National Academy of Sciences \\ of Ukraine, Kharkiv, Ukraine; \\ ${ }^{2}$ V.N. Karazin Kharkiv National University, Kharkiv, Ukraine; \\ ${ }^{3}$ Kharkiv National University of Radioelectronics, Kharkiv, Ukraine \\ E-mail:yuriyaverkov@gmail.com;prokopen@ire.kharkov.ua;sh47@ukr.net; \\ yavm@ire.kharkov.ua
}

In this work, the excitation problem of bulk-surface helicons by a point magnetic dipole moving in a vacuum parallel to the element of magnetized solid-state plasma cylinder is theoretically studied. The external magnetic field is directed parallel to the cylinder axis. The problem is solved in the magnetostatic approximation. It is shown that hybrid modes of the magnetic type with large values of the azimuthal mode index and one field variation along the radius are most efficiently excited at nonrelativistic velocities of magnetic dipole.

PACS: 03.50.De, 52.35.-g

\section{INTRODUCTION}

The determination of the generation mechanisms of electromagnetic waves due to a movement of charged particles in various electrodynamic systems is an actual problem of radiophysics and electronics. The waves of millimeter and submillimeter wavelengths are of particular interest. They are actively used in biology [1], medicine [2], for the transmission of electromagnetic signals in the earth's atmosphere [3], for the implementation of broadband wireless communication [4], in submillimeter spectroscopy [5] and other applications of science and technology.

In this regard, it is of interest to study the properties of magnetoplasma waves of the special type that exist at the boundary between a magnetoactive plasma of semiconductor and a dielectric (vacuum). Such waves are surface and bulk-surface helicons [6 - 10]. These waves exist independently of the ratio between the signal frequency and the collision frequency of charge carriers in classical and quantizing magnetic fields. The magnetic field components of a helicon are large compared to the electric field components. Therefore, for example, coupled helicon-spin surface waves can appear at the semiconductor-ferrite interface [11]. Another important feature of helicon waves is their relatively low phase velocity. The excitation of surface helicons by a magnetic dipole moving above flat surface of a semi-bounded semiconductor in an external magnetic field was theoretically studied in [12].

Note that helicon waves continue to be of interest both for fundamental research on the properties of materials and for applied research.

Thus, in [13], the theory of helicon waves propagating in three-dimensional Weyl semimetals, the conductivity of which is determined by the topological properties of the wave functions of charge carriers, was presented. In [14], the collisionless Landau damping of helicon waves propagating in a Dirac semimetal placed in a quantizing magnetic field was predicted. In particu- lar, the effect of giant oscillations of the damping coefficient of helicons was predicted. In [15], the study of helicons in semimetals was extended to the case of a pseudomagnetic background field. A qualitative experimental facility was proposed for observing helicons with magnetic and pseudomagnetic background fields.

In this work, the excitation of bulk-surface helicons by a point magnetic dipole moving near the lateral surface of semiconductor cylinder in a coaxial external magnetic field is theoretically studied in the magnetostatic approximation. The expression of energy losses of a magnetic dipole for the excitation of helicon eigenmodes of the cylinder is obtained, and the numerical analysis of magnitudes of these losses is carried out for modes with different values of the azimuthal and radial mode indices.

\section{PROBLEM STATEMENT AND BASIC EQUATIONS}

Consider a cylindrical plasma solid-state waveguide with a radius $\rho_{c}$ that occupies the space region of $0 \leq \rho \leq \rho_{c}, 0 \leq \varphi \leq 2 \pi$, and $|z| \leq \infty$. The waveguide is located in a vacuum in an external constant magnetic field, the intensity vector $\vec{H}_{0}$ of which is directed parallel to its axial symmetry axis. We assume that the plasma has a high conductivity of $n$-type. The equilibrium density of plasma electrons is $N_{0}$. The positively charged background of the crystal lattice of solid-state plasma compensates the negative charge of conduction electrons. In a vacuum a point magnetic dipole moves with a constant longitudinal velocity $v_{0}<<c$ (where $c$ is the velocity of light in vacuum) at the distance $\rho_{m}>\rho_{c}$ from the cylinder axis. The magnetic moment $\vec{M}$ of the dipole is given by the following expression:

$$
\begin{gathered}
\vec{M}(\vec{r}, t)=\vec{M}_{0} \delta\left(x-x_{0}\right) \delta\left(y-y_{0}\right) \delta\left(z-v_{0} t\right), \\
\vec{M}(\vec{r}, t)=\left(0,0, M_{z}(\vec{r}, t)\right),
\end{gathered}
$$


where $\vec{M}_{0}$ is the constant component of the magnetic moment, $\delta(x)$ is the Dirac delta function, $x_{0}$ and $y_{0}$ are the coordinates of the dipole in the plane $(x, y)$. In the approximation of a point dipole we can consider the circular current created by a charged particle (macroparticle) rotating in a plane $(x, y)$, provided that the Larmor radius $r_{L}$ of this particle is less than the distance $\rho_{m}-\rho_{c}$. From the condition $r_{L} \ll \rho_{m}$ it is possible to obtain the condition for the magnitude of the transverse velocity component $v_{\perp}$ of a charged particle perpendicular to the external magnetic field. To do this, we use the definition of the Larmor radius $r_{L}=v_{\perp} /\left|\Omega_{H}\right|$, where $\Omega_{H}=Q H_{0} / m_{q} c$ is the Larmor frequency of a charged particle with charge $Q$ and mass $m_{q}$. Then the point dipole approximation will be valid under the condition $v_{\perp} \ll<\rho_{m}\left|\Omega_{H}\right|$. The constant longitudinal component of the magnetic moment $M_{0 z}$ of such the circular current will be determined by the expression [16]:

$$
M_{0 z}=\frac{m_{q} v_{\perp}^{2}}{2 H_{0}} .
$$

The current density created by such a dipole will be determined by the expression [16]:

$$
\vec{j}_{m}(\vec{r}, t)=c \operatorname{rot} \vec{M}(\vec{r}, t) \text {. }
$$

The electric $\vec{E}^{m}(\vec{r}, t)$ and magnetic $\vec{H}^{m}(\vec{r}, t)$ fields of the point dipole at an arbitrary space point $\vec{r}$ in an instant time $t$ satisfy the following equations:

$$
\begin{gathered}
\operatorname{rot} \vec{H}^{m}(\vec{r}, t)=\frac{4 \pi}{c} \vec{j}_{m}(\vec{r}, t), \\
\operatorname{rot} \vec{E}^{m}(\vec{r}, t)=-\frac{1}{c} \frac{\partial}{\partial t} \vec{H}^{m}(\vec{r}, t), \\
\operatorname{div} \vec{H}^{m}(\vec{r}, t)=0 .
\end{gathered}
$$

In equation (5), due to the small value of the electric field, the displacement current is not taken into account.

Free fields (radiation fields) $\vec{E}^{v}(\vec{r}, t)$ and $\vec{H}^{v}(\vec{r}, t)$ in a vacuum satisfy the equations of magnetostatics:

$$
\begin{aligned}
& \operatorname{rot} \vec{H}^{v}(\vec{r}, t)=0, \\
& \operatorname{div} \vec{H}^{v}(\vec{r}, t)=0 .
\end{aligned}
$$

Note, the considered magnetostatic approximation is valid if the radiation wavelength $\lambda=2 \pi c / \omega$ (in a vacuum) is substantially greater than the cylinder radius [17], it is equivalent to

$$
\omega<<\frac{c}{\rho_{c}} .
$$

In this case, the fields found from equations (8) and (9) will be correct at distances from the cylinder, which is less than the wavelength $\lambda$.

Free fields $\vec{E}^{c}(\vec{r}, t)$ and $\vec{H}^{c}(\vec{r}, t)$ in the solid-state plasma cylinder satisfy the quasi-stationary Maxwell equations and the motion equation of electrons in a plasma-like medium:

$$
\operatorname{rot} \vec{H}^{c}(\vec{r}, t)=\frac{4 \pi}{c} \vec{j}_{c}(\vec{r}, t),
$$

$$
\begin{gathered}
\operatorname{div} \vec{H}^{c}(r, t)=0, \\
\operatorname{rot} \vec{E}^{c}(\vec{r}, t)=-\frac{1}{c} \frac{\partial}{\partial t} \vec{H}^{c}(\vec{r}, t), \\
\vec{E}^{c}(\vec{r}, t)+\frac{1}{c}\left[\vec{u}(\vec{r}, t) \times \vec{H}_{0}\right]=0,
\end{gathered}
$$

where $\vec{u}(\vec{r}, t)$ and $\vec{j}_{c}(\vec{r}, t)=e N_{0} \vec{u}(\vec{r}, t)$ are the velocity and the density of current of charge carriers (electrons) in a plasma-like medium, respectively.

The solutions of the above equations will be carried out by the method of separation of variables representing all magnitude variables in the form of a set of spacetime harmonics. For example, the magnetic field strength is determined by the formula:

$$
\begin{aligned}
& \vec{H}(\vec{r}, t)=\sum_{n=-\infty}^{\infty} \int_{-\infty}^{\infty} \int_{-\infty}^{\infty} \vec{H}_{n}\left(\rho, q_{z}, \omega\right) \times \\
& \times \exp \left[i\left(q_{z} z+n \varphi-\omega t\right)\right] d q_{z} d \omega,
\end{aligned}
$$

where $\omega, q_{z}$ and $n$ are the frequency, and the longitudinal wavenumber and the spatial harmonic number (coinciding with the azimuthal mode index), respectively; $i^{2}=-1$. Note, the considered quasi-stationary approximation for describing fields in a plasma-like medium is valid if this medium is well conducting, i.e. the smallness condition of frequency of the fields $\omega$ in comparison with the collision frequency of electrons $v$ is satisfied:

$$
\omega<<v \text {. }
$$

In this case, it is justified to neglect the displacement current in comparison with the conduction current of electrons in a plasma-like medium [17]. In addition, the magnitude of the constant magnetic field is assumed such to be condition

$$
v<<\omega_{H},
$$

where $\omega_{H}=e H_{0} / m c$ is the Larmor frequency of plasma electrons; $e$ and $m$ are the charge and effective mass of electrons of the plasma-like medium. The fulfillment of conditions (16) and (17) is necessary for the existence of bulk-surface helicons in the considered solid-state plasma waveguide (see $[8,19,11]$ ), the excitation of which we consider. In the vacuum region, the field distributions of such waves have a monotonically decreasing character with distance from the cylinder surface in the radial direction, and in the cylinder region, they have an oscillating character in the radial direction. Note that due to the fulfillment of conditions (16) and (17) the motion equation of electrons (14) in a plasma-like medium is correct. This corresponds to the situation when the force lines of the magnetic field are "frozen" into the electron liquid, and the electrons are "placed" on these force lines [11]. Physically, this means that the considered plasma-like medium is ideally conducting, and the alternating surface current flows along the cylinder surface in the direction of its generating lines [9]. A consequence of the ideal conductivity of plasma-like medium is the equality to zero of the longitudinal components of free electric fields $E_{z}$ in both the medium and the vacuum. Nonzero components of free fields are $E_{\rho}, E_{\varphi}$ for the electric field, and $H_{\rho}$, $H_{\varphi}, H_{z}$ for the magnetic field. 
The boundary conditions on the cylinder surface (when $\rho=\rho_{c}$ ) are the conditions for the continuity of the $H_{\rho}$ and $H_{z}$ components of resultant magnetic field (dipole and radiation fields):

$$
\begin{aligned}
& \left.H_{\rho}^{m}(\vec{r}, t)\right|_{\rho=\rho_{c}}+\left.H_{\rho}^{v}(\vec{r}, t)\right|_{\rho=\rho_{c}}=\left.H_{\rho}^{c}(\vec{r}, t)\right|_{\rho=\rho_{c}}, \\
& \left.H_{z}^{m}(\vec{r}, t)\right|_{\rho=\rho_{c}}+\left.H_{z}^{v}(\vec{r}, t)\right|_{\rho=\rho_{c}}=\left.H_{z}^{c}(\vec{r}, t)\right|_{\rho=\rho_{c}} .
\end{aligned}
$$

The component $H_{\varphi}$ of the resultant magnetic field at the boundary $\rho=\rho_{c}$ undergoes the discontinuity due to the presence of the surface current with the density $j_{z}^{s}(z, t)$ :

$$
\begin{aligned}
& \left.H_{\varphi}^{m}(\vec{r}, t)\right|_{\rho=\rho_{c}}+\left.H_{\varphi}^{v}(\vec{r}, t)\right|_{\rho=\rho_{c}}- \\
& -\left.H_{\varphi}^{c}(\vec{r}, t)\right|_{\rho=\rho_{c}}=\frac{4 \pi}{c} j_{z}^{s}(z, t) .
\end{aligned}
$$

Our goal is to find the fields created by the point dipole and reflected from the cylinder surface (radiation field) from the above-given equations and boundary conditions. Obviously, the radiation field is expressed through the parameters of the point dipole and the medium. Then we shall determine the energy losses of the point dipole for the excitation of bulk-surface helicons per time unit as (see [12])

$$
\frac{d W_{e}}{d t}=-M_{0 z} \frac{\partial H_{z}^{v}\left(\vec{r}_{m}, t\right)}{\partial t}
$$

where the value of the time derivative of magnetic field strength component $H_{z}^{v}(\vec{r}, t)$ is taken at the point $\vec{r}=\vec{r}_{m}$, where the magnetic dipole is located.

Using the integral representations of the Dirac delta functions (see [18]) and substituting them into the righthand side of (1), we obtain the following expression for the component $M_{z}$ of the point magnetic dipole in the cylindrical coordinate system:

$$
\begin{aligned}
& M_{z}(\rho, \varphi, z, t)=\frac{M_{0 z}}{(2 \pi)^{2}} \sum_{n=-\infty}^{\infty} \int_{0}^{\infty} k J_{n}(k \rho) J_{n}\left(k \rho_{m}\right) d k \times \\
& \times \int_{-\infty}^{\infty} \exp \left\{i\left[q_{z} z+n\left(\varphi-\varphi_{m}\right)\right]\right\} \delta\left(\omega-q_{z} v_{0}\right) d q_{z},
\end{aligned}
$$

where $J_{n}(u)$ is the Bessel function of the $n$-th order of the first kind [18]; $\varphi_{m}$ is the azimuthal coordinate of the point dipole; the variable of integration $k$ is the wavenumber of field spatial harmonic of the point dipole.

Let us find the expressions for the components of magnetic field strength created by the point dipole. Applying the operation of calculating the rotor to the left and right sides of equation (5) and using the definition of current (4), we obtain the following equation:

$$
\begin{aligned}
& \Delta \vec{H}^{m}(\rho, \varphi, z, t)=-4 \pi[\text { graddiv } \vec{M}(\rho, \varphi, z, t)- \\
& \left.-\Delta \vec{M}^{m}(\rho, \varphi, z, t)\right],
\end{aligned}
$$

where

$$
\begin{aligned}
& \left(\Delta \vec{H}^{m}\right)_{\rho}=\Delta H_{\rho}^{m}-\frac{H_{\rho}^{m}}{\rho^{2}}-\frac{2}{\rho^{2}} \frac{\partial H_{\varphi}^{m}}{\partial \varphi}, \\
& \left(\Delta \vec{H}^{m}\right)_{\varphi}=\Delta H_{\varphi}^{m}-\frac{H_{\varphi}^{m}}{\rho^{2}}+\frac{2}{\rho^{2}} \frac{\partial H_{\rho}^{m}}{\partial \varphi},
\end{aligned}
$$

$$
\begin{gathered}
\left(\Delta \vec{H}^{m}\right)_{z}=\Delta H_{z}^{m}, \\
\Delta=\Delta_{\perp}+\frac{\partial^{2}}{\partial z^{2}}=\frac{1}{\rho} \frac{\partial}{\partial \rho} \rho \frac{\partial}{\partial \rho}+\frac{1}{\rho^{2}} \frac{\partial^{2}}{\partial \varphi^{2}}+\frac{\partial^{2}}{\partial z^{2}}, \\
(\Delta \vec{M})_{\rho}=0 ;(\Delta \vec{M})_{\phi}=0 ;(\Delta \vec{M})_{z}=\Delta M_{z}, \\
\operatorname{grad}_{\rho} \operatorname{div} \vec{M}^{m}(\rho, \varphi, z, t)=\frac{\partial^{2} M_{z}^{m}(\rho, \varphi, z, t)}{\partial \rho \partial z} \\
\operatorname{grad}_{\varphi} \operatorname{div} \vec{M}^{m}(\rho, \varphi, z, t)=\frac{1}{\rho} \frac{\partial^{2} M_{z}^{m}(\rho, \varphi, z, t)}{\partial \varphi \partial z} \\
\operatorname{grad}_{z} \operatorname{div} \vec{M}^{m}(\rho, \varphi, z, t)=\frac{\partial^{2} M_{z}^{m}(\rho, \varphi, z, t)}{\partial z^{2}}
\end{gathered}
$$

From equation (23), taking into account (28) and (31), we obtain the equation for the field component $H_{z}^{m}(\rho, \varphi, z, t)$ :

$$
\Delta H_{z}^{m}(\rho, \varphi, z, t)=4 \pi \Delta_{\perp} M_{z}^{m}(\rho, \varphi, z, t) .
$$

Let us represent the Fourier component $H_{z}^{m}\left(\rho, q_{z}, \omega\right)$ of the magnetic field strength created by the magnetic dipole in the form

$$
H_{z}^{m}\left(\rho, q_{z}, \omega\right)=\int_{0}^{\infty} k J_{n}(k \rho) H_{z}^{m}\left(k, q_{z}, \omega\right) d k .
$$

From equation (32), taking into account (15), (22), (26), (28), and (33), we obtain the following expression for $H_{z}^{m}\left(k, q_{z}, \omega\right)$ :

$$
\begin{aligned}
& H_{z}^{m}\left(\rho, q_{z}, \omega\right)=\frac{M_{0 z}}{\pi} \times \\
& \times \int_{0}^{\infty} d k \frac{k^{3} J_{n}\left(k \rho_{m}\right) J_{n}(k \rho)}{k^{2}+q_{z}^{2}} \delta\left(\omega-q_{z} v_{0}\right) .
\end{aligned}
$$

To find the field components $H_{\rho}^{m}(\rho, \varphi, z, t)$ and $H_{\varphi}^{m}(\rho, \varphi, z, t)$ we introduce new dependent variables:

$$
H_{ \pm}(\rho, \varphi, z, t)=H_{\rho}(\rho, \varphi, z, t) \pm i H_{\varphi}(\rho, \varphi, z, t),
$$

for which the wave equations can be written:

$$
\begin{aligned}
& \Delta H_{ \pm}(\rho, \varphi, z, t)-\frac{H_{ \pm}(\rho, \varphi, z, t)}{\rho^{2}} \pm \frac{2 i}{\rho^{2}} \frac{\partial H_{ \pm}(\rho, \varphi, z, t)}{\partial \varphi}= \\
& =-4 \pi \frac{\partial}{\partial z}\left[\frac{\partial M_{z}^{m}(\rho, \varphi, z, t)}{\partial \rho} \pm \frac{i}{\rho} \frac{\partial M_{z}^{m}(\rho, \varphi, z, t)}{\partial \varphi}\right] .
\end{aligned}
$$

Substituting expressions (15), (22), (33) into (36) and using definitions (24), (25), (35), we obtain solutions for $H_{\rho}^{m}(\rho, \varphi, z, t)$ and $H_{\phi}^{m}(\rho, \varphi, z, t)$ in the form

$$
\begin{aligned}
& H_{\rho}^{m}\left(\rho, q_{z}, \omega\right)=\frac{2 i q_{z} M_{0 z}}{\pi} \times \\
& \times \int_{0}^{\infty} d k \frac{k^{2} J_{n}\left(k \rho_{m}\right) J_{n}^{\prime}(k \rho)}{k^{2}+q_{z}^{2}} \delta\left(\omega-q_{z} v_{0}\right), \\
& H_{\varphi}^{m}\left(\rho, q_{z}, \omega\right)=-\frac{2 q_{z} n M_{0 z}}{\pi \rho} \times \\
& \times \int_{0}^{\infty} d k \frac{k J_{n}\left(k \rho_{m}\right) J_{n}(k \rho)}{k^{2}+q_{z}^{2}} \delta\left(\omega-q_{z} v_{0}\right),
\end{aligned}
$$

where the prime near the Bessel function means its derivative with respect to the argument.

Let us find the free fields of the considered magnetized cylindrical solid-state plasma waveguide. Applying the operation of calculating the rotor from the left and 
right sides of equation (8) and using the equation (9), we obtain the wave equation for the component $H_{z}^{v}(\rho, \varphi, z, t)$ of the radiation field in the vacuum:

$$
\Delta_{\perp} H_{z}^{v}(\rho, \varphi, z, t)=0 .
$$

Substituting the expression (15) into (39), we obtain the solution for $H_{z}^{v}\left(\rho, q_{z}, \omega\right)$ in the form

$$
H_{z}^{v}\left(\rho, q_{z}, \omega\right)=C_{1} K_{n}\left(\left|q_{z}\right| \rho\right),
$$

where $K_{n}(u)$ is the modified Bessel function of the $n$-th order of the second kind (Macdonald function) [18]; $C_{1}$ is the arbitrary constant. Using solution (40), from the equation (9) we find expressions for the components $H_{\rho}^{v}\left(\rho, q_{z}, \omega\right)$ and $H_{\phi}^{v}\left(\rho, q_{z}, \omega\right)$ :

$$
\begin{aligned}
& H_{\rho}^{v}\left(\rho, q_{z}, \omega\right)=\frac{n}{q_{z} \rho} H_{z}^{v}\left(\rho, q_{z}, \omega\right), \\
& H_{\varphi}^{v}\left(\rho, q_{z}, \omega\right)=\frac{1}{i q_{z}} \frac{\partial H_{z}^{v}\left(\rho, q_{z}, \omega\right)}{\partial \rho} .
\end{aligned}
$$

Determining the rotor from the left and right sides of the motion equation of electron (14) and using the equation (11), we obtain the equation for the magnetic field of helicon wave in the cylinder:

$$
\frac{\partial}{\partial z} \operatorname{rot} \vec{H}(\rho, \varphi, z, t)=\frac{4 \pi e N}{c H_{0}} \frac{\partial \vec{H}(\rho, \varphi, z, t)}{\partial t} .
$$

Taking into account (15), solutions of the equation (43) have the following form

$$
\begin{aligned}
& H_{\rho}^{c}\left(\rho, q_{z}, \omega\right)=\frac{q}{\kappa^{2}} \times \\
& \times\left[\frac{i q_{z}}{q} \frac{\partial H_{z}^{c}\left(\rho, q_{z}, \omega\right)}{\partial \rho}-\frac{i n}{\rho} H_{z}^{c}\left(\rho, q_{z}, \omega\right)\right], \\
& H_{\varphi}^{c}\left(\rho, q_{z}, \omega\right)=\frac{1}{\kappa^{2}} \times \\
& \times\left[q \frac{\partial H_{z}^{c}\left(\rho, q_{z}, \omega\right)}{\partial \rho}-\frac{n q_{z}}{\rho} H_{z}^{c}\left(\rho, q_{z}, \omega\right)\right], \\
& H_{z}^{c}\left(\rho, q_{z}, \omega\right)=C_{2} J_{n}(\kappa \rho),
\end{aligned}
$$

where $q=4 \pi e \omega N_{0} / q_{z} c H_{0}$ is the wavenumber of helicons and $\kappa=\sqrt{q^{2}-q_{z}^{2}}$ is the transverse wavenumber of the wave of helicon origin; $C_{2}$ is the arbitrary constant.

Substituting expressions for the fields of the magnetic dipole and free fields in the both vacuum and cylinder in (18) and (19), and taking into account the definition (15), we obtain the following expression for the constant $C_{1}$ :

$$
\begin{aligned}
& C_{1}=\frac{1}{K_{n}\left(\left|q_{z}\right| \rho_{c}\right) \Delta_{0}\left(q_{z}, \omega\right)} \times \\
& \times\left[\frac{q}{\kappa^{2}}\left(\frac{n}{\rho_{c}}-\frac{q_{z} \kappa}{q} \frac{J_{n}^{\prime}\left(\kappa \rho_{c}\right)}{J_{n}\left(\kappa \rho_{c}\right)}\right) H_{z}^{m}\left(\rho_{c}, q_{z}, \omega\right)-\right. \\
& \left.-i H_{\rho}^{m}\left(\rho_{c}, q_{z}, \omega\right)\right],
\end{aligned}
$$

where

$$
\Delta_{0}\left(q_{z}, \omega\right)=\frac{q_{z}}{\left|q_{z}\right|} \frac{K_{n}^{\prime}\left(\left|q_{z}\right| \rho_{c}\right)}{K_{n}\left(\left|q_{z}\right| \rho_{c}\right)}+\frac{q_{z}}{\kappa} \frac{J_{n}^{\prime}\left(\kappa \rho_{c}\right)}{J_{n}\left(\kappa \rho_{c}\right)}-\frac{n q}{\rho_{c} \kappa^{2}} \text {. }
$$

In order to use formulas (15), (21), (40), and (47), (48) to calculate the energy losses of the point dipole for the excitation of bulk-surface helicons, it is first necessary to calculate the integrals in the expressions for the components of the magnetic dipole fields $H_{\rho}^{m}\left(\rho_{c}, q_{z}, \omega\right)$ and $H_{z}^{m}\left(\rho_{c}, q_{z}, \omega\right)$. Using the corresponding formulas from [19], we obtain the expressions for the required components of the magnetic dipole field in the form

$$
\begin{aligned}
& H_{\rho}^{m}\left(\rho, q_{z}, \omega\right)=\frac{2 i q_{z} M_{0 z}}{\pi} \delta\left(\omega-q_{z} v_{0}\right) \times \\
& \times\left\{\begin{array}{l}
\left|q_{z}\right| I_{1}\left(\left|q_{z}\right| \rho_{c}\right) K_{0}\left(\left|q_{z}\right| \rho_{m}\right), \quad n=0, \\
\frac{\rho_{c}^{n-1}}{2 \rho_{m}^{n}}+\frac{\left|q_{z}\right|}{2}\left[I_{n-1}\left(\left|q_{z}\right| \rho_{c}\right)+\right. \\
\left.+I_{n+1}\left(\left|q_{z}\right| \rho_{c}\right)\right] K_{n}\left(\left|q_{z}\right| \rho_{m}\right), \quad n>0,
\end{array}\right. \\
& H_{z}^{m}\left(\rho, q_{z}, \omega\right)=-\frac{q_{z}^{2} M_{0 z}}{\pi} \delta\left(\omega-q_{z} v_{0}\right) \times \\
& \times I_{n}\left(\left|q_{z}\right| \rho_{c}\right) K_{n}\left(\left|q_{z}\right| \rho_{m}\right),
\end{aligned}
$$

where $I_{n}(u)$ is the modified Bessel function of the $n$-th order of the first kind (Infeld function) [18].

When calculating the energy losses of the point dipole upon integration over $d q_{z}$, we introduce into consideration small dissipative losses and use the Sokhotskii formula (see [20]) to go around the pole of $\Delta_{0}\left(q_{z}, \omega\right)=0$ :

$$
\lim _{\gamma \rightarrow 0} \frac{1}{x-i \gamma}=i \pi \delta(x)+\frac{P}{x}
$$

where $P$ is the main value of the integral; $\gamma$ is the small value of bypass radius around the pole $x$. The equation $\Delta_{0}\left(q_{z}, \omega\right)=0$ is the dispersion equation for the eigenwaves of cylinder, i.e. the dispersion equation for bulk-surface helicons when $\kappa^{2}>0$.

In final, the expressions for the energy losses of the point dipole for the excitation of space-time harmonics with numbers $n$ have the following form

$$
\frac{d W_{0 e}}{d t}=-\frac{M_{0 z}^{2} \omega_{0}}{v_{0}} K_{0}\left(\left|q_{z}\right| \rho_{m}\right) \frac{A_{0}\left(q_{z 0}, \omega_{0}\right)}{\left|B_{0}\left(q_{z 0}, \omega_{0}\right)\right|},
$$

when $n=0$,

$$
\frac{d W_{p e}^{( \pm)}}{d t}=-\frac{M_{0 z}^{2} \omega_{0}}{v_{0}} K_{p}\left(\left|q_{z}\right| \rho_{m}\right) \frac{A_{p}^{( \pm)}\left(q_{z 0}, \omega_{0}\right)}{\left|B_{p}^{( \pm)}\left(q_{z 0}, \omega_{0}\right)\right|},
$$

when $n \neq 0$, where

$$
\begin{aligned}
& A_{0}\left(q_{z 0}, \omega_{0}\right)=q_{z 0}\left|q_{z 0}\right| \frac{K_{0}\left(\left|q_{z 0}\right| \rho_{m}\right)}{K_{0}\left(\left|q_{z 0}\right| \rho_{c}\right)} \times \\
& \times\left[\frac{\left|q_{z 0}\right|}{\kappa_{0}} \frac{J_{0}^{\prime}\left(\kappa_{0} \rho_{c}\right)}{J_{0}\left(\kappa_{0} \rho_{c}\right)} I_{0}\left(\left|q_{z 0}\right| \rho_{c}\right)+2 I_{1}\left(\left|q_{z 0}\right| \rho_{c}\right)\right], \\
& A_{p}^{( \pm)}\left(q_{z 0}, \omega_{0}\right)=-q_{z 0} \frac{K_{p}\left(\left|q_{z}\right| \rho_{m}\right)}{K_{0}\left(\left|q_{z}\right| \rho_{c}\right)} \times \\
& \times\left[\frac{q_{z 0} q_{0}}{\kappa_{0}^{2}}\left( \pm \frac{p}{\rho_{c}}-\frac{q_{z 0} \kappa_{0}}{q_{0}} \frac{J_{p}^{\prime}\left(\kappa_{0} \rho_{c}\right)}{J_{p}\left(\kappa_{0} \rho_{c}\right)}\right) I_{p}\left(\left|q_{z 0}\right| \rho_{c}\right)-\right. \\
& \left.-2\left(\frac{\rho_{c}^{p-1}}{\rho_{m}^{p}}+\left|q_{z 0}\right|\left(I_{p-1}\left(\left|q_{z 0}\right| \rho_{c}\right)+I_{p+1}\left(\left|q_{z 0}\right| \rho_{c}\right)\right)\right)\right],
\end{aligned}
$$




$$
\begin{gathered}
B_{0}\left(q_{z 0}, \omega_{0}\right)=\left.\frac{\partial \Delta_{0}\left(q_{z}, \omega\right)}{\partial \omega}\right|_{\substack{n=0 \\
\omega=\omega_{0} \\
q_{z}=q_{z 0}}}, \\
B_{p}^{( \pm)}\left(q_{z 0}, \omega_{0}\right)=\left.\frac{\partial \Delta_{0}\left(q_{z}, \omega\right)}{\partial \omega}\right|_{\substack{n= \pm p \\
\omega=\omega_{0} \\
q_{z}=q_{z 0}}},
\end{gathered}
$$

where $p=|n| \geq 1$, and signs $( \pm)$ mean cases $n>0$ and $n<0$, respectively; the quantities $q_{z 0}=\omega_{0} / v_{0}$ and $\omega_{0}$ satisfy the Vavilov-Cherenkov resonance condition and correspond to the roots of the dispersion equation $\Delta_{0}\left(q_{z}, \omega\right)=0 ; q_{0}$ and $\kappa_{0}$ are the wavenumbers of helicon waves calculated when $q=q_{z 0}$ and $\omega=\omega_{0}$.

\section{NUMERICAL ANALYSIS OF ENERGY LOSSES OF THE MAGNETIC DIPOLE}

We performed the numerical analysis of the energy losses of the magnetic dipole for the excitation of bulksurface helicons using the following dimensionless quantities:

$$
\Phi_{0}=\frac{1}{P_{0}} \frac{d W_{0 e}}{d t} ; \Phi_{p}^{( \pm)}=\frac{1}{P_{0}} \frac{d W_{p e}^{( \pm)}}{d t} ; \beta=\frac{v_{0}}{c},
$$

where $P_{0}=\omega_{c}^{2} M_{0 z}^{2} / \rho_{c}^{2} v_{0}$. Semiconductor indium antimonide with electronic conductivity $(n-\mathrm{InSb})$, in which $N_{0}=1.35 \cdot 10^{14} \mathrm{~cm}^{-3}$, was chosen as the material of the cylinder. The strength of the external magnetic field was specified $H_{0}=8 \mathrm{kGs}$, the radius of the cylinder $\rho_{c}=0.25 \mathrm{~cm}$, and the distance between the magnetic dipole and the cylinder axis $\rho_{m}=0.5 \mathrm{~cm}$.

Figs. 1-3 show the values of the magnetic dipole energy losses (in units $P_{0}$ ) for the excitation of bulksurface helicon modes with azimuthal indices $n$ for various values of the dimensionless dipole velocity $\beta$.

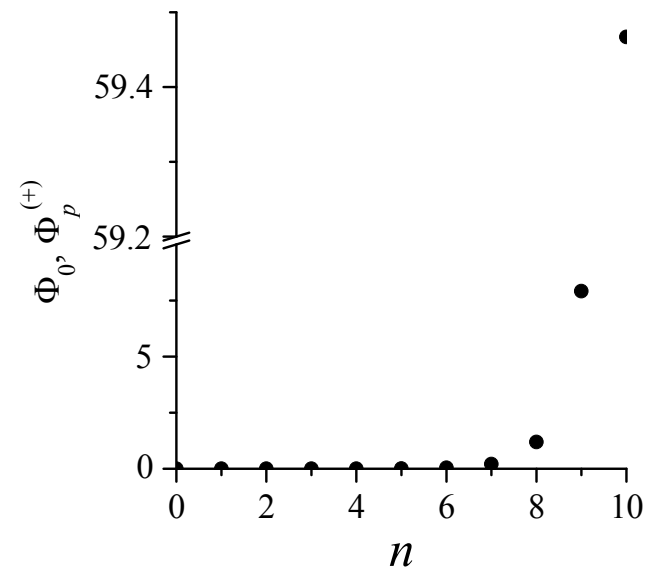

Fig. 1. Energy losses of the point magnetic dipole for the excitation of bulk-surface helicons of the $H_{01}$ - and $E H_{n 1}$-type when $\beta=0.1$

From Fig. 1 it can be seen when $\beta=0.1$ the values of losses increase monotonically with increasing $n$. In this case, modes with one field variation along the radius $(s=1)$ are only excited.

In Fig. 2 modes $H_{01}$ and $E H_{n 1}$ are shown with black dots, and mode $E H_{12}$ is shown with the empty circle. From Fig. 2 it can be seen when $\beta=0.2$ the dependence of the value of losses on $n$ ceases to be monotonic. Local maxima are appeared, and when $n>>1$ the loss values are decreased by an order of magnitude than when $\beta=0.1$ (see Figs. 1 and 2). In addition to the modes with $n \geq 0$, the mode with $n=-1$ and $s=1$ is excited, and when $n=1$, modes with one $(s=1)$ and two $(s=2)$ field variations along the radius are simultaneously excited. In addition, the analysis showed that the magnitude of the losses $\Phi_{1}^{(-)}$ for the excitation of mode $E H_{-11}$ exceeds the magnitude one $\Phi_{1}^{(+)}$for the excitation of mode $E H_{11}$.

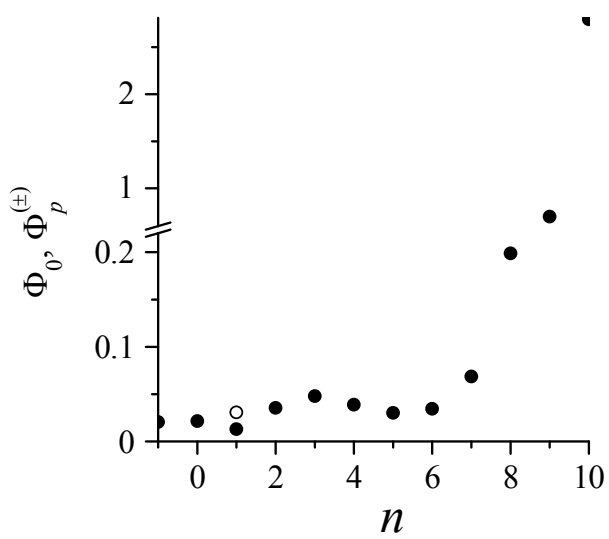

Fig. 2. Energy losses of the point magnetic dipole for the excitation of bulk-surface helicons of the $H_{01}$ - and $E H_{n \text { s }}$-type when $\beta=0.2$

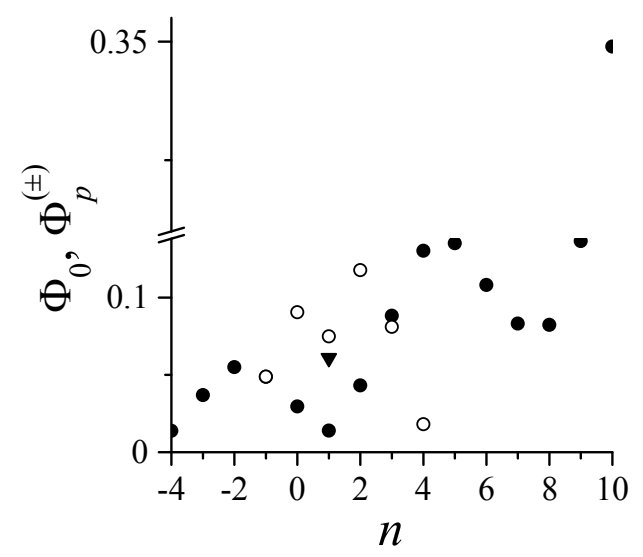

Fig. 3. Energy losses of the point magnetic dipole for the excitation of bulk-surface helicons of the $H_{0 s}$ - and $E H_{n \text { s }}$-type when $\beta=0.3$

In Fig. 3 modes $H_{01}$ and $E H_{n 1}$ are shown with black dots, modes $H_{02}$ and $E H_{\mathrm{n} 2}$ are shown with empty circles, and mode $\mathrm{EH}_{13}$ is shown with the black triangle. From Fig. 3 it can be seen when $\beta=0.3$ the quantities of excited modes with $s=2$ increases and mode $E H_{13}$ appears. When the dipole velocity $\beta=0.3$ the values of its energy losses for the excitation of identical modes decrease by $\sim 60$ and $\sim 1000$ times in comparison with $\beta=0.2$ and $\beta=0.1$, respectively.

From the numerical analysis, it follows that for $n \geq 0$ the values of the losses for the excitation of modes $E H_{\mathrm{n} 2}$ exceed the values of the losses for the 
excitation of modes $E H_{n 1}$, in which $n=0,1,2$. When $n=3$ these values become comparable in magnitude, and when $n \geq 4$ the values of the losses for the excitation of modes $\mathrm{EH}_{\mathrm{n} 2}$ become less than the values of the losses for the excitation of modes $E H_{n 1}$. The value of the losses for the excitation of mode $E H_{-12}$ is comparable in magnitude with one for the excitation of mode $E H_{-11}$.

We also note that the values of the losses for the excitation of modes with the same values $s$ and the same magnitude, but different in sign values $n$ turn out to be different. This may indicate about the demonstration of the effect of nonreciprocity in the propagation of bulksurface helicons in a magnetized plasma cylinder. From the comparison of Figs. 1-3 it follows that the lower velocity of the magnetic dipole $\beta$, modes $E H_{n 1}$ with large values of the azimuthal mode index $n$ are more efficiently excited. In this case, modes $E H_{n \mathrm{~s}}$ with $s>1$ are not excited. The excitation of such modes becomes possible when $\beta$ increases.

\section{CONCLUSIONS}

In work, the problem of the excitation of bulksurface helicons by a point magnetic dipole moving in a vacuum near a magnetized solid-state plasma cylinder parallel to its generating lines is studied theoretically. The external magnetic field is directed parallel to the symmetry axis of the cylinder. A circular current created by a charged particle rotating in a plane perpendicular to the cylinder axis, provided the Larmor radius of this particle is less than the distance from the dipole to the lateral surface of the cylinder, is considered as a point dipole.

In a vacuum, the electromagnetic fields (radiation fields) satisfy the equations of magnetostatics, and in a cylinder, they (free fields) satisfy the quasi-stationary Maxwell equations and the motion equation of electrons in an ideally conducting plasma-like medium. The chosen approximation is valid if the radiation wavelength (in vacuum) is much greater than the radius of the cylinder, and the displacement current in the plasma cylinder is negligible in comparison with the conduction current due to the high conductivity of the plasma medium. The magnitude of the constant magnetic field was assumed such that the Larmor frequency of charge carriers significantly exceeds its collision frequency in the plasma cylinder. Under these conditions, the propagation of bulk-surface helicons is possible in the plasma. In addition, this physically means that an alternating surface current flows along the cylinder surface in the direction of its generating lines.

The consequence of the ideal conductivity of the plasma-like medium is the equality to zero of the longitudinal (parallel to the cylinder axis) components of free electric fields in both the medium and the vacuum. The boundary conditions on the cylinder surface are the conditions for the continuity of the radial and longitudinal components of alternating magnetic field strength. The axial component of magnetic field strength on the cylinder surface suffers a discontinuity due to the surface current.

The numerical analysis of the expression for the energy losses of the magnetic dipole for the excitation of bulk-surface helicons showed that when the velocity of the magnetic dipole is lower, modes $E H_{n 1}$ with large values of the azimuthal mode index $n$ are more efficiently excited. In this case, modes $E H_{n \mathrm{~s}}$ with $s>1$ are not excited. The excitation of modes $E H_{n \mathrm{~s}}$ with $s>1$ becomes possible when the velocities of the magnetic dipole $\beta \geq 0.2$. With an increase of the magnetic dipole velocity above $\beta=0.1$, the dependence of the magnitude of losses on the azimuthal mode index $n$ ceases to be monotonically-increasing. Local maxima appear on this dependence.

In addition, the values of the losses for the excitation of modes with the same values of the radial index $s$ and the same magnitude, but different in sign values of the azimuthal index $n$ turn out to be different. This indicates about the demonstration of the effect of nonreciprocity in the propagation of bulk-surface helicons with identical spatial field distributions in a magnetized plasma cylinder.

\section{REFERENCES}

1. E. Pickwell, B.E. Cole, A.J. Fitzgerald, et al. Simulation of terahertz pulse propagation in biological systems // Applied Physics Letters. 2004, v. 84, issue 12, p. 2190-2192.

2. P.C. Ashwort, E. Pickwell-MacPherson, E. Provenzano, et al. Terahertz pulsed spectroscopy of freshly excised human breast cancer // Optics Express. 2009, v. 17, issue 15, p. 12444-12454.

3. Y. Yang, A. Shutler, D. Grischkowsky. Measurement of the transmission of the atmosphere from 0.2 to $2 \mathrm{THz} / /$ Optics Express. 2011, v. 19, issue 9, p. $8830-8838$.

4. T. Nagatsuma, S. Horiguchi, Y. Minamikata, et al. Terahertz wireless communications based on photonics technologies // Optics Express. 2013, v. 21, issue 20, p. 23736-23747.

5. M.C. Kemp. Explosive detection by terahertz spectroscopy - a bridge too far // IEEE Transactions on Terahertz Science and Technology. 2011, v. 1, issue 1, p. 282-292.

6. S.I. Khankina, V.M. Yakovenko. Excitation of surface electromagnetic waves in semiconductors // Fizika Tverdogo Tela. 1967, v. 9, issue 2, p. 578-582 (in Russian).

7. S.I. Khankina, V.M. Yakovenko. On the theory of surface waves in solids // Fizika Tverdogo Tela. 1967, v. 9, issue 10, p. 29433-2947 (in Russian).

8. Yu.O. Averkov, Yu.V. Prokopenko, V.M. Yakovenko. Surface helicons in a structure with graphene monolayer lying on a 3D plasma $/ / 9^{\text {th }}$ International Kharkiv Symposium on Physics and Engineering of Microwaves, Millimeter and Submillimeter Waves (MSMW'2016), Kharkiv, Ukraine, 2016, p. 1-4.

9. Yu.O. Averkov, Yu.V. Prokopenko, V.M. Yakovenko. Helicons in plasma solid-state waveguide of cylindrical configuration // Problems of Atomic Sci- 
ence and Technology. Series: "Plasma Electronics and New Methods of Acceleration”. 2019, № 4, p. 19-23.

10. Yu.O. Averkov, Yu.V. Prokopenko, V.M. Yakovenko. Helicons in Solid-State Plasma of Cylindrical Configuration // IEEE Ukrainian Microwave Week $(U k r M W)$ Dnipro, Ukraine, 2020, DOI: 0.1109/UkrMW49653.2020.9252703.

11. N.N. Beletskii, V.M. Svetlichnyi, D.D. Khalameida, and V. M. Yakovenko. Electromagnetic Phenomena in the Microwave Region in Inhomogeneous Semiconductor Structures. Kiev: "Naukova Dumka", 1991, 216 p.

12. V.M. Yakovenko, I.V. Yakovenko. Surface wave excitation in solids by magnetic dipoles // Solid State Communications. 1985, v. 55, № 10, p. 911-913.

13. F.M.D. Pellegrino, M.I. Katsnelson, M. Polini. Helicons in Weyl semimetals // Physical Review B. 2015, v. 92, issue 20, p. 201407(R).

14. D.I. Yasnov, A.P. Protogenov, P.M. Echenique, E.V. Chulkov. Collective excitations and universal broadening of cyclotron absorption in Dirac semi- metals in a quantizing magnetic field // Physical Review B. 2020, v. 101, issue 24, p. 245206(7).

15.E.V. Gorbar, V.A. Miransky, I.A. Shovkovy, P.O. Sukhachov. Pseudomagnetic helicons // Physical Review B. 2017, v. 95, issue 11, p. 115422(15).

16. J.D. Jackson. Classical Electrodynamics. New York: "John Wiley and Sons, Inc.", 1999, 808 p.

17. L.D. Landau, E.M. Lifshitz. Course of Theoretical Physics, v. 8: Electrodynamics of Continuous Media. M.: "Nauka", 1984, 621 p.

18. G.A. Korn, T.M. Korn. Mathematical handbook for scientists and engineers. Definitions, Theorems, and Formulas for Reference and Review. New York: "Dover publications, Inc. Mineola", 1984, 832 p.

19. A.P. Prudnikov, Yu.A. Brychkov, O.I. Marichev. Integrals and series: special functions. M.: "Nauka", 1986, $756 \mathrm{p}$.

20. V.S. Vladimirov. Equations of Mathematical Physics. New York: "Marcel Dekker, Inc.", 1871, 426 p.

Article received 26.05.2021

\section{ПОТЕРИ ЭНЕРГИИ ТОЧЕЧНОГО МАГНИТНОГО ДИПОЛЯ ПРИ ВЗАИМОДЕЙСТВИИ С ЗАМАГНИЧЕННЫМ ПЛАЗМЕННЫМ ЦИЛИНДРОМ}

\section{Ю.О. Аверков, Ю.В. Прокопенко, А.А. Шматько, В.М. Яковенко}

Теоретически изучена задача о возбуждении объемно-поверхностных геликонов точечным магнитным диполем, движущимся в вакууме параллельно образующим замагниченного твердотельного плазменного цилиндра. Внешнее магнитное поле направлено параллельно оси цилиндра. Задача решена в магнитостатическом приближении. Показано, что гибридные моды магнитного типа с большими значениями азимутального модового индекса и с одной вариацией полей по радиусу наиболее эффективно возбуждаются при нерелятивистских скоростях магнитного диполя.

\section{ВТРАТИ ЕНЕРГІї ТОЧКОВОГО МАГНІТНОГО ДИПОЛЯ ВНАСЛІДОК ВЗАЕМОДІї ІЗ ЗАМАГНІЧЕНИМ ПЛАЗМОВИМ ЦИЛІНДРОМ}

\section{Ю.О. Аверков, Ю.В. Прокопенко, О.О. Шматько, В.М. Яковенко}

Теоретично вивчено задачу про збудження об'ємно-поверхневих геліконів точковим магнітним диполем, що рухається у вакуумі паралельно твірним замагніченого твердотільного плазмового циліндра. Зовнішнє магнітне поле направлено паралельно осі циліндра. Задача розв'язана в магнітостатичному наближенні. Показано, що гібридні моди магнітного типу з великими значеннями азимутального модового індексу і $з$ однією варіацією поля вздовж радіуса найбільш ефективно збуджуються в разі нерелятивістських швидкостей магнітного диполя. 